\title{
Signature Graphs for Effective Localization
}

\author{
Sven Rönnbäck Tomas Berglund
}

Håkan Fredriksson Kalevi Hyyppä

\author{
Division of EISLAB \\ Department of Computer Science \\ and Electrical Engineering \\ Luleå University of Technology \\ SE-97187 LULEÅ, SWEDEN \\ Email: $\{$ sr,tb,hf, kalevi $\} @$ csee.ltu.se
}

\begin{abstract}
We present a novel method for the localization of an autonomous vehicle in two dimensions. Instead of focussing on properties of obstacles, we emphasize the description of freespace. We introduce the concept of signature graphs which is a compact data structure describing available free-space of an environment. It therefore also represents a map of the environment.
\end{abstract}

A signature is an edge in the graph and is defined by seven parameters. An edge indicates that it is possible for the vehicle to move between two graph nodes.

The signature graph is flexible and can easily be updated as new information about the environment is collected.

We have with an autonomous wheelchair implemented our methods and tests them with successful results.

\section{INTRODUCTION}

${ }^{1}$ This paper addresses the problem of localization of a vehicle in two dimensions. This problem is important in areas of research and applications such as assistive robotics and industrial mobile robots.

An efficient method for localization gives great benefits and can be used, for instance, for effective navigation [1], [2], [3]. Much research in the area of vehicle localization has already been made [4], [5], [6], [7]. One of the main drawbacks of many methods are the association problems involved. The modeling or representation of different geometric structures, with various complexity, such as points, circles, lines, etc is involved in many solutions [7][8].

We propose a novel and efficient method of solving the problem of efficient localization. Instead of trying to model obstacles, we model the free-space, i.e. space where the vehicle can go free from obstacles.

Given an environment, we build a data structure called a signature graph. The signature graph is built from signatures and it allows efficient computation; for instance the localization of a vehicle.

Voronoi based graphs can be used for representation of routes[9][10].

For improving the efficiency of using autonomous vehicles, such as the wheelchair, we propose methods for localization by introducing what we denote as a signature. We show how

\footnotetext{
${ }^{1 *}$ This work was supported in part by the EU program Interreg IIIA Nord
}

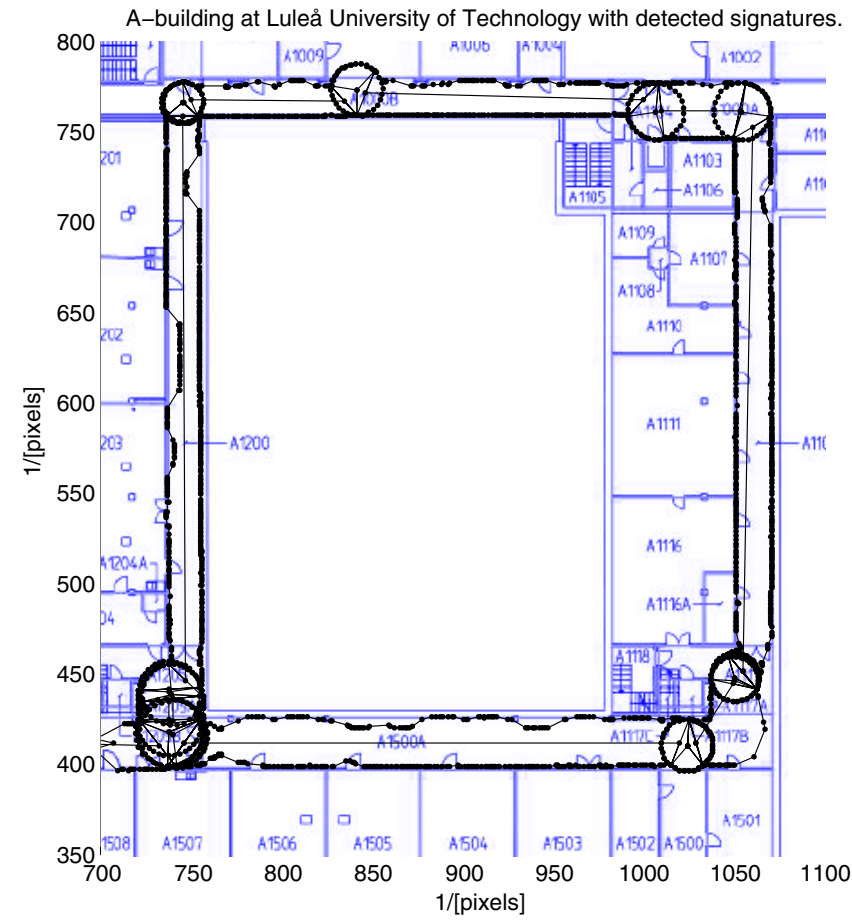

Fig. 1. Signatures plotted on a construction plan. The circles represents nodes in the signature graph. Visible differences between the signatures and the map are caused by obstacles found in the corridors such as benches and tables. The signatures span up the free-space available for the wheelchair. It is possible to see that the door to room A1505 is partly opened, check with Figure 11.

these signatures can be connected to build a signature graph, visible in Figure 1. We show that signatures and signature graphs give effective localization.

A signature is an edge in a signature graph and is matched against other signatures with seven parameters. These parameters are uniquely defined by circle sectors in the local freespace. An edge of the graph is telling that it is possible for the vehicle to move between the two nodes in the graph.

The main advantage of using a signature graph for localization compared to earlier presented localization methods is that it efficiently parameterizes the free-space available 
for navigation. Furthermore, the signature graph can be used for more purposes than efficient localization. Our proposed methods and data structures are suitable for different kinds of optimization and for visualization of data.

We present definitions of signatures and the signature graph. Different methods, benefits and, possibilities for the use of signature graphs are proposed. An implementation that produces signature graphs has been implemented.

The data structure and algorithms were successfully tested in a real-life environment using our semi-autonomous wheelchair.

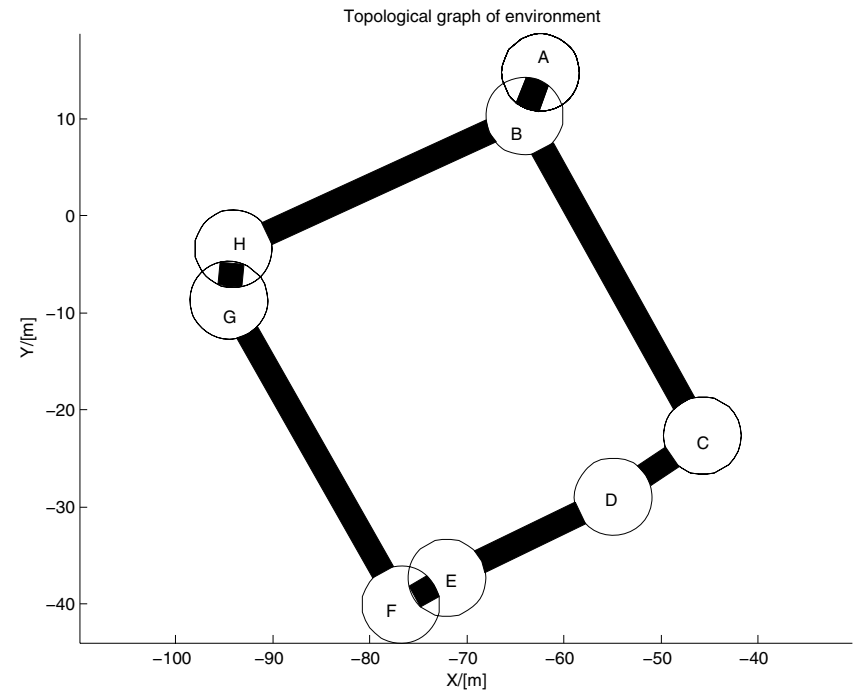

Fig. 2. A topological metric graph of A-building at Luleå University of Technology. The graph has eight nodes, $\{A, \ldots, H\}$ and seven edges.

\section{The Signature Graph}

The underlying idea is to compactly represent available freespace for a vehicle.

They are detected as uniquely defined identifiers in two dimensions. These signatures are connections between two circle chords.

A signature marks the possibility to move between two graph nodes is used to define an edge in the signature graph.

A signature or node is taken to build a vertex of the graph $G$ and free space between two nodes is taken to build an edge in the graph, see Figure 2 and compare it to Figure 1. Therefore, a signature graph $G=(V, E)$ consists of nodes or vertices $V=\{A, B, \ldots, H\}$ (circle sectors) and edges $E$ (free-space).

Graph theory is a wide research area of its own [11]. There are numerous efficient ways of using, representing, and computing with graphs [12], [13].

By representing an environment as a signature graph, we have the possibility to use powerful algorithms from graph theory research area.

\section{A. Definition of a signature}

A signature is defined the seven parameters, see Figure 3.

$$
\text { signaure }=\left\{r_{1}, r_{2}, d, \gamma_{1}, \gamma_{2}, c_{1}, c_{2}\right\} .
$$

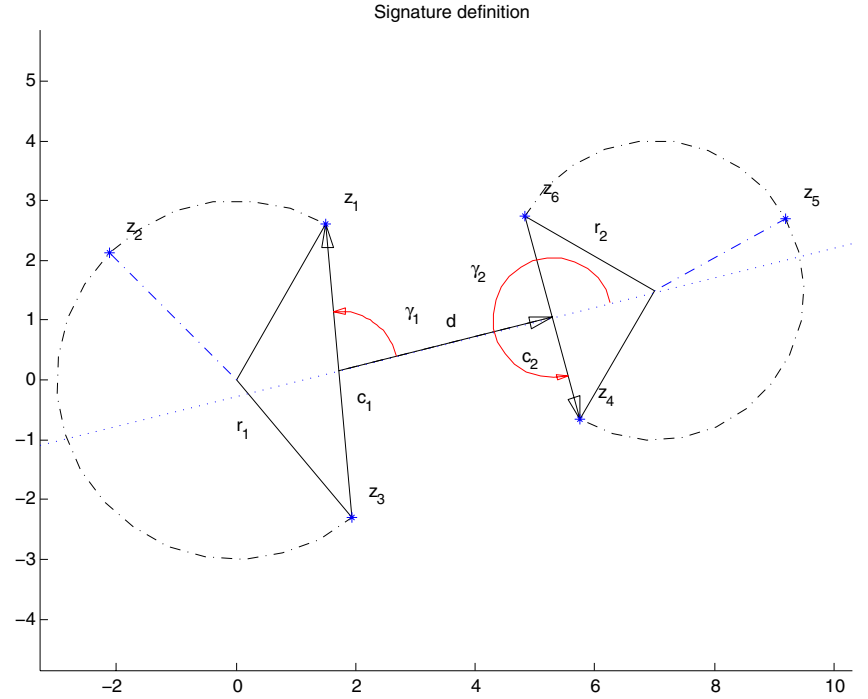

Fig. 3. The definition of a signature is based on the angles between the chord vectors and the vector connecting the two circle sectors. The chord lengths and the radius of the circle sectors are also contained in the definition.

The radii of the two circles $\left\{r_{1}, r_{2}\right\}$, the two chord lengths $\left\{c_{1}, c_{2}\right\}$, the chord angles relative to the axis of the signature, $\left\{\gamma_{1}, \gamma_{2}\right\}$, the length $\left\{d=\left|z_{d}\right|\right\}$ and the length of the two circle chords $c_{1}$ and $c_{2}$,

$$
c_{1}=\left|z_{1}-z_{3}\right|, \quad c_{2}=\left|z_{4}-z_{6}\right| .
$$

The frame attached to the signature has its origin at $\left(z_{1}+\right.$ $\left.z_{3}\right) / 2$. The length $d$ of a signature is defined as the distance between the mid points of the circle chords,

$$
z_{d}=\frac{\left(z_{4}+z_{6}\right)-\left(z_{1}+z_{3}\right)}{2}, \quad d=\left|z_{d}\right| .
$$

The angles of the chords relative to the $\hat{X}$-axis of the signature are

$$
\gamma_{1}=\angle\left(z_{1}-z_{3}\right) \bar{z}_{d}, \quad \gamma_{2}=\angle\left(z_{4}-z_{6}\right) \bar{z}_{d}
$$

\section{B. Matching of signatures}

When a signature is defined and a signature graph is built, it is necessary to have an efficient tool for the localization of a vehicle which detects new signatures from range data collected by the scanning laser.

Signatures are identified from environmental data collected by a range scanning laser. Figure 5 shows a detected signature together with the laser range data. A found signature is matched against reference signatures for effective localization. A found signature is matched to reference signature, stored in a database, by the Mahalanobis distance [14]. Let $X_{s}$ be a vector with the seven signature parameters.

$$
X_{s}=\left[\begin{array}{lllllll}
r_{1} & r_{2} & c_{1} & c_{2} & \gamma_{1} & \gamma_{2} & d
\end{array}\right]
$$

Let $Z_{s}$ be the a vector that has the observed signature properties and $X_{s}(n)$ be the known properties of signature $n$. Innovation covariance $S_{s}(n)$ is calculated as

$$
S_{s}(n)=E\left[X_{s}(n) X_{s}(n)^{T}\right]+E\left[Z_{s} Z_{s}^{T}\right] .
$$




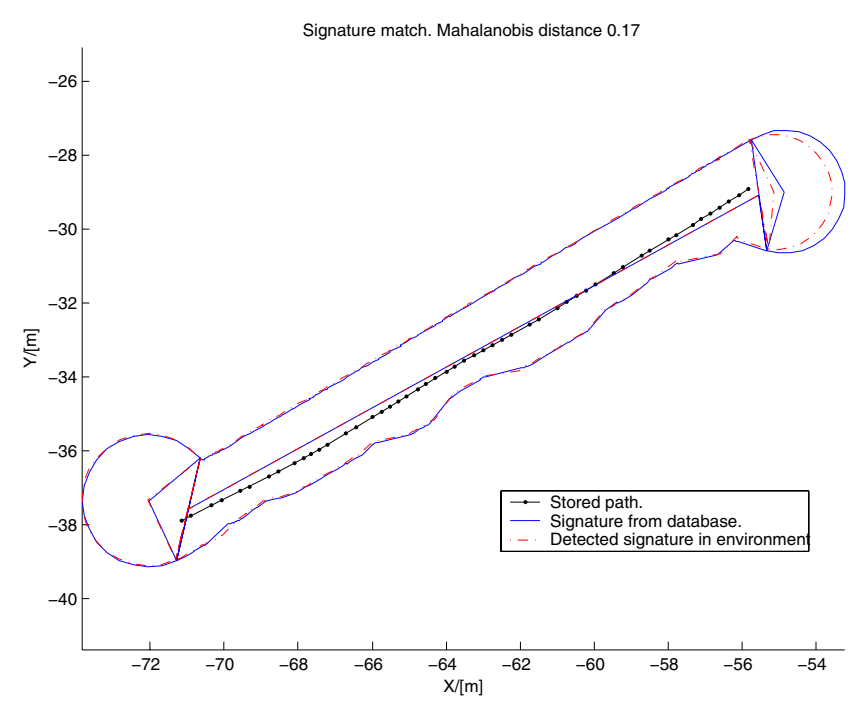

Fig. 4. A signature, plotted with a solid line, was detected from collected range data. It was matched against reference signatures, stored in a signature graph, using the Mahalanobis distance. The reference signature is plotted dashed. Different trajectories can be stored in the signature object; one is plotted as the dotted solid line.

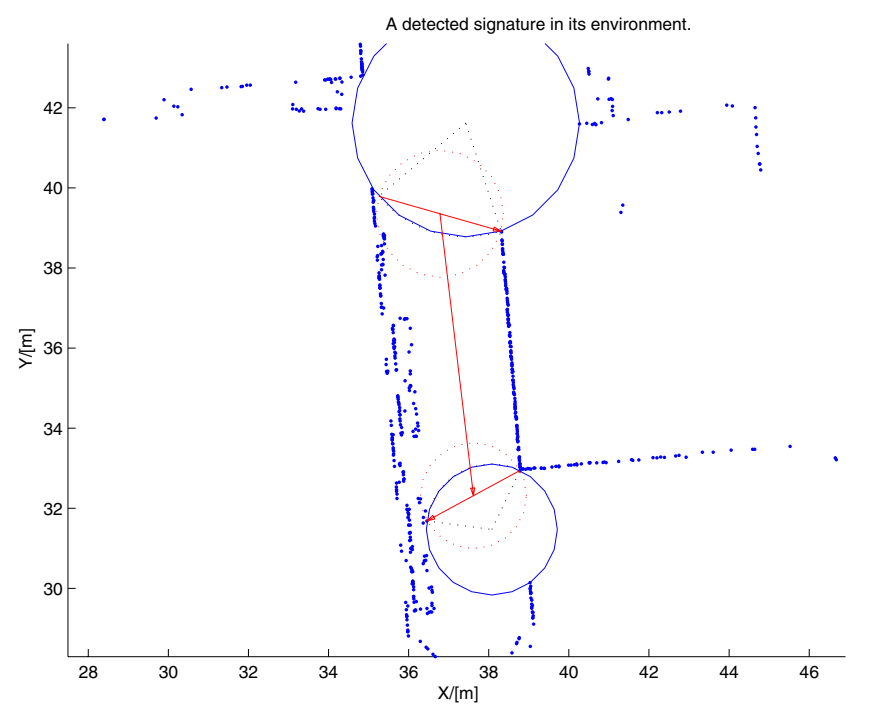

Fig. 5. A detected signature plotted in its environment. It consists of two circle chords and a connecting line. A signature indicates that there is exists free-space for navigation.

The Mahalanobis distance,

$$
\nu_{s i g}(n)=\left(Z_{s}-X_{s}(n)\right)^{T} S_{s}(n)^{-1}\left(Z_{s}-X_{s}(n)\right),
$$

, which is $\chi^{2}$ distributed as it is assumed the noise involved are normally distributed. The distance variable $\nu_{\text {sig }}(n)$ is also dimensionless and compensates for interactions between the variables. We take the match, $\nu_{s i g}(n)$, with highest probability and check against tables if the match was significant. The test has seven degrees of freedom and is equal to the number of observed signature parameters.

The confidence level of the $\chi^{2}$ distribution to a given degrees of freedom is given in statistical tables.
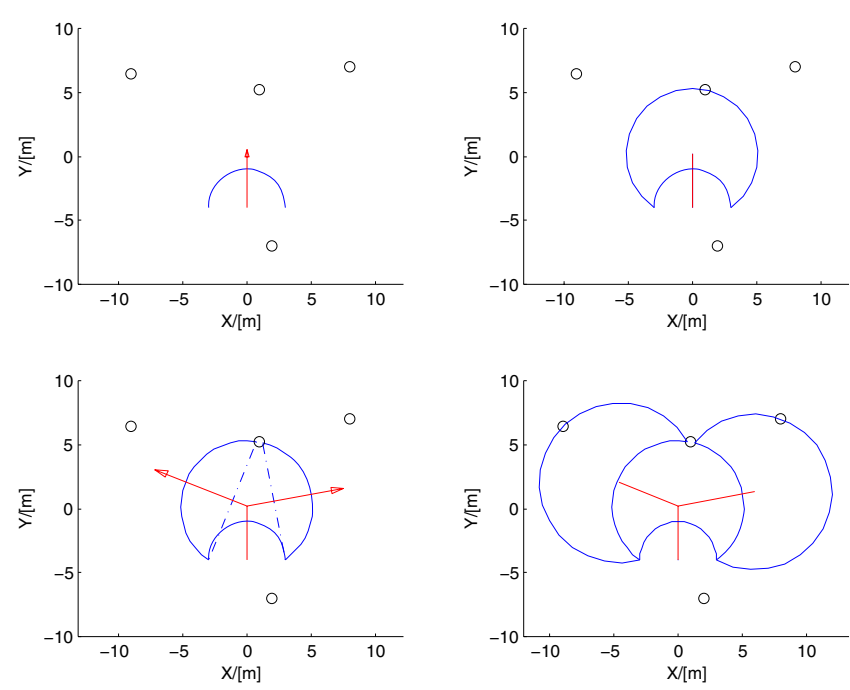

Fig. 6. A thee step expansion sequence by the circle sector expansion method. The arrows marks are expanding directions. The connected circle centers form a Voronoi diagram.

\section{The Circle Sector Expansion method}

To find signatures we use expanding circle sectors, see Figure 6. The circle sector expansion (CSE) method is intuitive and used to calculate a reduced Voronoi diagram based on two dimensional data [15]. The main idea with the method is to expand circle sectors until they are bounded by data.

It creates a chain of consecutive circle sectors that describe the available free-space for navigation. The centers of the consecutive circles form a path used as an initial path for the vehicle.

Figure 6 shows the first three steps of an circle sector expansion sequence in an environment with four measurements marked with small circles.

The circle sector in sub-figure one marks the initial circle sector. In each expansion step the sector is divided by a measurement into two new circle sectors. The arrows attached to the chords show the expanding directions.

Each circle sector $k$ defined by three measurements $z_{1}(k), z_{2}(k), z_{3}(k)$. Parameters $\theta_{c}(k)$ marks the heading of the circle sector, the sector angle $\beta(k)$ and the circle center $\left[x_{c}(k), y_{c}(k)\right]^{T}$. Circle $k$ is has the parameters:

$$
\operatorname{cir}_{c}(k)=\left(x_{c}(k), y_{c}(k), r_{c}(k), \beta_{c}(k), \theta_{c}(k)\right) .
$$

The complex number $z_{v}$ is used to calculate the circle center,

$$
z_{v}(k)=\left[z_{1}(k)-z_{2}(k)\right] \overline{\left[z_{3}(k)-z_{2}(k)\right]},
$$

where $\overline{\left[z_{3}(k)-z_{2}(k)\right]}$ is the complex conjugate of $\left[z_{3}(k)-\right.$ $\left.z_{2}(k)\right]$. The derived analytic expression for the circle sector center $z_{c}(k)$ is

$z_{c}(k)=\frac{z_{1}(k)+z_{3}(k)}{2}+\frac{\left[z_{v}(k)+\overline{z_{v}(k)}\right]\left[z_{1}(k)-z_{3}(k)\right] e^{i \pi / 2}}{2\left[z_{v}(k)-\overline{z_{v}(k)}\right]}$.

The radius is $r_{c}(k)=\left|z_{c}(k)-z_{1}(k)\right|$. The circle sector arc is divided into two new sectors by measurement $z_{2}(k)$. 


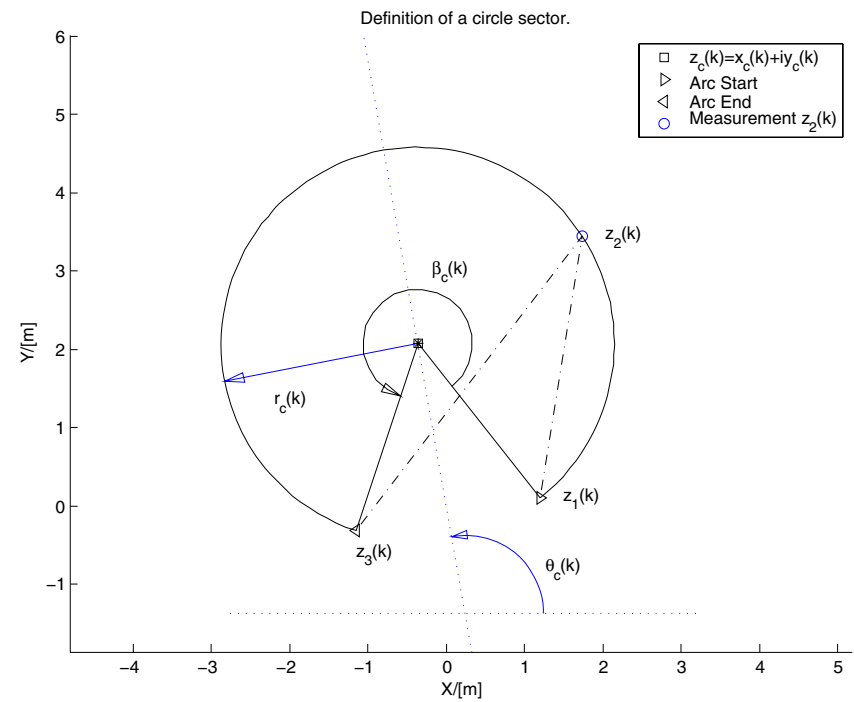

Fig. 7. The circle sector is defined by the three complex numbers $z_{1}(k), z_{2}(k), z_{3}(k)$. The center of the circle sector is $z_{c}(k)=x_{c}+i y_{c}$. The radius is $r_{c}(k)$ and the sector angle $\beta_{c}(k)$. The heading of the sector is $\theta_{c}(k)$.

When a sector is bounded it can either, based on the chord length, stop the expansion or be divided into two new circle sectors.

It is possible for our wheelchair to drive in the environment if the chord length is more than $0.8 \mathrm{~m}$; then the circle sector expansion will continue. If chord length is less then $0.8 \mathrm{~m}$ the circle sector expansion will stop. If the circle is not bounded by any data the radius is set to $10 \mathrm{~m}$.

Figure 8 shows a typical corridor environment with present obstacles. The circle sector expansion method is used to find a path through the environment.

The initial vehicle path is the dotted solid line created by connecting the circle centers. It is smoothed with a Bezier curve [16].

It is also possible to calculate a safety margin from the circle sectors. In Figure 8 they are plotted as dashed lines on each side of the polygon created by connecting the circle centers. The safety boundaries are useful to check if there is a risk for the vehicle to collide with the environment or any obstacle.

\section{IMPLEMENTATION AND LOCALIZATION USING SIGNATURE GRAPHS}

The algorithms and data structures described in previous section are implemented in the Java programming language. The Java implementation is executed in MATLAB[17]. Our code is tested using a wheelchair for performing different tasks, namely moving between signatures.

To evaluate the implementation, the algorithms, and data structures, we use an autonomous wheelchair, Figure 9. As range sensor we use a slightly pitch mounted range scanning laser of model SICK LMS200 [18]. The pitch angle is useful for covering the whole height span from floor to sensor placement.

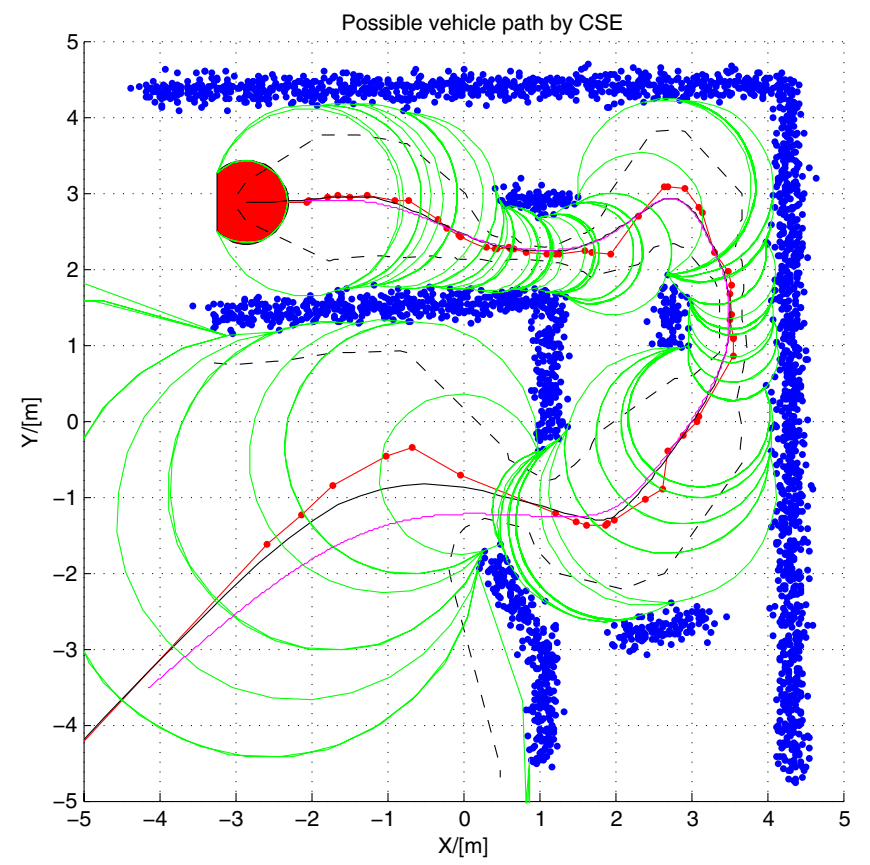

Fig. 8. An example of the circle sector expansion in an artificial corridor environment with present obstacles. The CSE method finds a possible path pass the obstacles. In this case the strategy for the expansion process was to always pick the circle sector with the longest chord.

With the laser, the vehicle is able to gather about 1700 twodimensional data points per second (at $4.7 \mathrm{~Hz}$ ) with a viewing angle of $180^{\circ}$ and within a range of about $4 \mathrm{~m}$ before the laser beam hits the floor. Points positioned less than $0.1 \mathrm{~m}$ from the the floor are filtered away and the remaining points are projected onto the floor.

By this, we assume that there are no obstacles, stairs etc, lower than $0.1 \mathrm{~m}$ from the floor. Furthermore, the vehicle carries a fiber optic rate gyro, KVH-Ecore 1000, and wheelencoders. It can be driven semi-autonomously or fully autonomously. We consider it to be a good representative for a broad range of sensor equipped autonomous vehicles.

\section{A. Selected test environment}

Underground corridors at Luleå University of Technology are used test environment, Figure 1. This environment plotted by measured laser scans together with computed signatures is shown in Figure 5. The chosen environment involves corridors, straight and non-straight crossings, and corners.

\section{B. Navigation between two signatures}

We have built a signature graph of the underground environment, Figure 1. We want to use the signature graph in order to move the vehicle between two signatures. Doing this, the vehicle in turn has to localize itself using the signature graph and its signatures.

Until the vehicle can localize itself at the next signature, the vehicle has to traverse edges, or free-space between consecutive signatures. If properties of the environment along these edges are unknown to the vehicle or if they are changing 


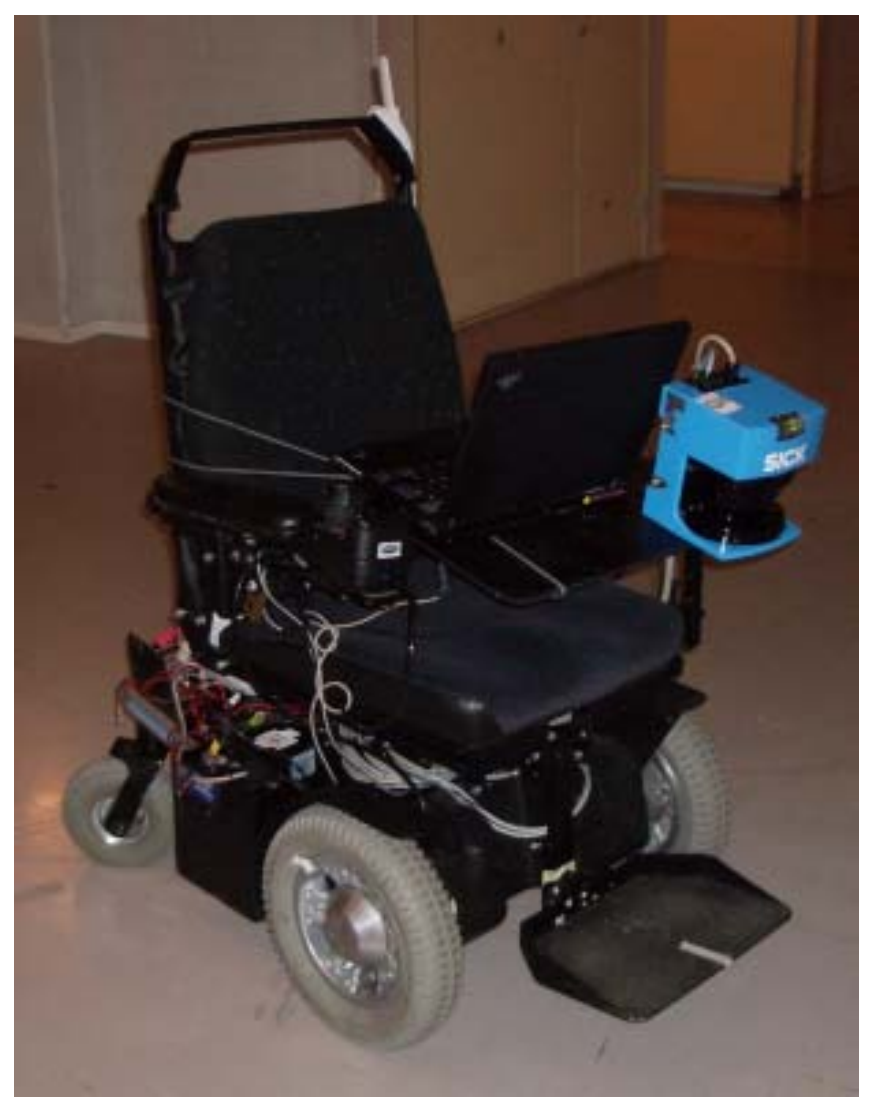

Fig. 9. We used a modern wheelchair equipped with a SICK laser for the tests. The laser is pitched down approximately $4^{\circ}$. It is also equipped with an embedded PC computer that runs a software that collects and time stamps sensor data readings. The computer is accessible over W-LAN, the antenna is visible on the top of the wheelchair. The used fiber optic gyro is visible under the seat between the front and back wheel.

with time, the vehicle has to work with on-line methods while traversing free space. Figure 10 shows online exploration using the circle sector expansion method.

In sub figure 1 the wheelchair, represented by a small half circle, enters an intersection. Collected data by the range scanning laser are plotted near the vehicle. The most recent trajectory made by the vehicle is plotted as a dotted solid line.

The circle sector expansion method is used to find paths in the data. A new possible paths to the south east is plotted as solid line from the vehicle position. The circle expansion process is stopped if the center of a new circle sector passes the solid boundary line.

In sub figure 2 a signature detected in collected range data is plotted and successfully matched against known signatures stored in a signature graph. The value of the Mahalanobis distance $\nu_{s i g}(n)$ is found in the title. The start of the detected signature is plotted as the half circle to the right in sub figure 1.

Free-space and safety boundaries calculated from the consecutive circle sectors expanded in collected range data are shown in sub figure 3 .
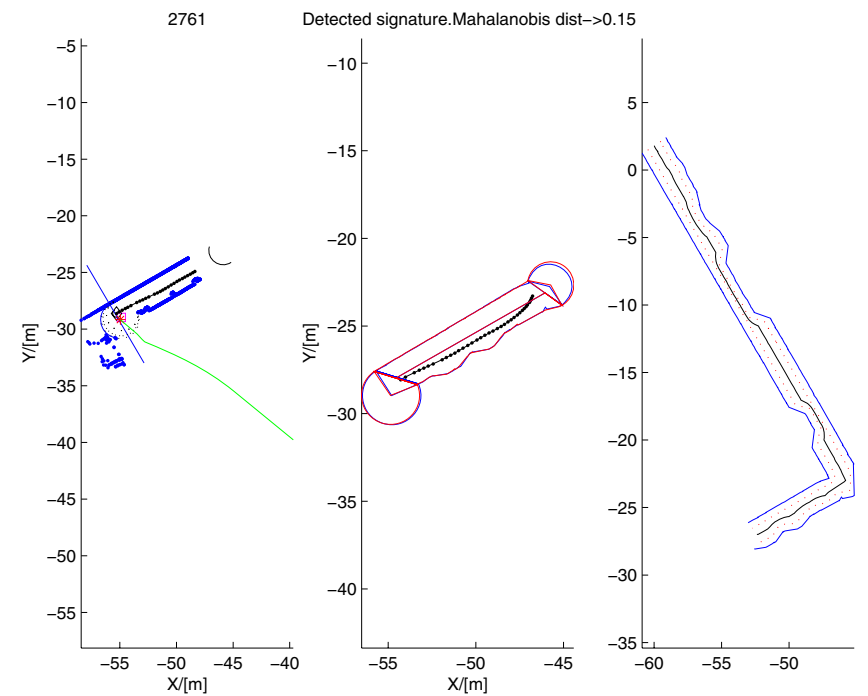

Fig. 10. 1) The vehicle in exploring mode. The small half circle is the wheelchair and the dotted solid line behind it is the trajectory. 2) The last detected signature that was successfully matched against reference signatures. 3) Estimated free-space reconstructed from information stored in signatures.

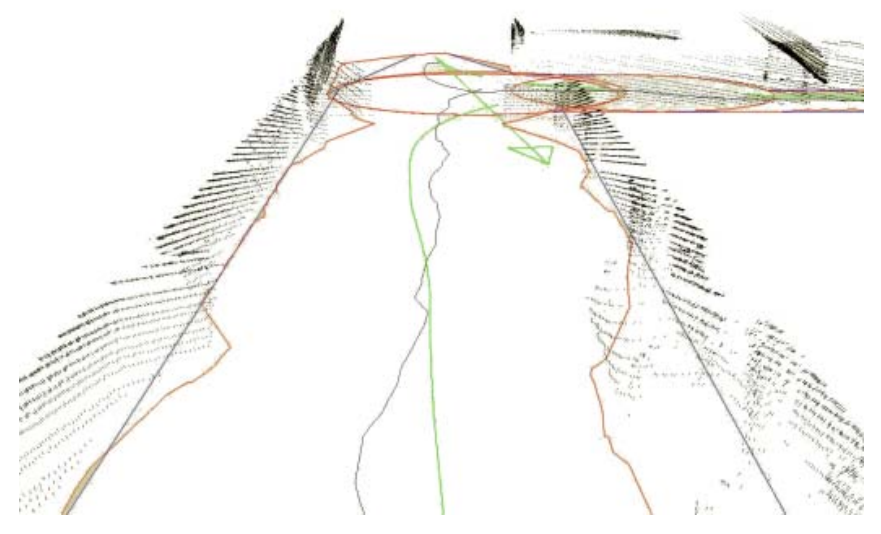

Fig. 11. A picture grabbed from the 3D software with the signature environment plotted. The wheelchair trajectory is visible as the smooth line near the center. The centered line that follows the contours of the walls is created by connecting the centers of the consecutive circles found in the expansion process. On the left side a partly opened door to room A1505 is visible.

\section{Visualization for understanding of data}

The Open Scene Graph 3D engine[19] is used to visualize results from tests, Figure 11. The location of the grabbed scene is in the corridor between room A1504 and A1505, see Figure 1. The collected laser scanner data are visible as the dotted strips on the walls. A partly opened door to room A1505 is visible to the left and a bench to the right.

The trajectory made by the wheelchair is the smooth line plotted on floor level. The centered line that follows the contours of the walls is created by the circle sector expansion. The wall contours are calculated from the consecutive 
circle sectors. The solid straight lines are estimates of walls positions.

The arrow visible near the corner marks a used coordinate system.

\section{CONCLUSIONS}

We have presented a novel method for localization of an autonomous vehicle in two dimensions. Instead of focussing on properties of obstacles, we emphasize the description of free-space. We have introduced the concept free-space signatures using the method of circle sector expansion (CSE) to find the signatures and use them to build a signature graph. A signature graph is a data structure suitable for describing free-space of an environment.

The uniquely defined signatures are edges of the graph and the connection between signatures are the nodes. An edge announces that there is enough free space for a vehicle to move between two graph nodes. The graph can be used for fast computations such as finding the shortest or fastest path to a desired location.

We have successfully implemented our proposed methods and data structures. Our implementations and ideas are tested in real-life using an autonomous wheelchair equipped with a scanning laser. The wheelchair is originally constructed as an aid for elderly or impaired people with special needs. The test environment was chosen such that it contained general surroundings such as corridors, crossings and obstacles.

Using the wheelchair in the chosen environment, we are able to efficiently build and use a signature graph as a representation of the environment. The vehicle we able localizes itself using the signature graph.

\section{FUTURE WORK}

There are lots of questions raised and interesting issues discovered during the work behind this paper.

First of all, we have presented a method of compacting the description of an environment into a signature graph. This is indeed a graph and graph theory is a wide and interesting research area.

It would be interesting to see whether and how it would be possible to use more than one vehicle at the same time for exploring an unknown environment and for building a signature graph. The vehicles must then be able to communicate and share signature and graph information.

One approach could then be to benefit wheelchair users in their daily life where the wheelchairs automatically exchange information as they meet. The information could consist of preferred vehicle paths, desired speed and angular velocity, and updates of a signature graphs used for localization and navigation.

\section{ACKNOWLEDGEMENT}

Thanks Patrik Emilsson, at Permobil AB, for his support and his help with the wheelchair. This work was supported by EU Interreg IIIA Nord.

\section{REFERENCES}

[1] T. Bailey, "Mobile robot localization and mapping in extensive outdoor environments," Ph.D. dissertation, Australian Centre for Field Robotics. Department of Aerospace, Mechanical and Mechatronic Engineering. University of Sydney, 2002.

[2] B. Lisien, D. Morales, D. Silver, G. Kantor, I. M. Rekleitis, and H. Choset, "The hierarchical atlas," IEEE Transactions on Robotics and Automation, vol. 21, no. 3, pp. 473-481, June 2005.

[3] J. E. Guivant, "Efficient simultaneous localization and mapping in large environments," Ph.D. dissertation, University Of Sydney, May 2002.

[4] H. Choset and K. Nagatani, "Topological simultaneous localization and mapping (slam): toward exact localization without explicit localization," IEEE Transactions on Robotics and Automation, vol. 17, no. 2, 2001.

[5] E. Acar, H. Choset, and J. Lee, "Sensor-based coverage with extended range detectors," IEEE Transactions on Robotics and Automation, vol. 22, no. 1, Feb 2006.

[6] P. Beeson, N. K. Jong, and B. Kuipers, "Towards autonomous topological place detection using the extended voronoi graph," in IEEE International Conference on Robotics and Automation (ICRA'05), 2005.

[7] P. Newman, "On the structure and solution of the simultaneous localisation and map building problem," Ph.D. dissertation, Australian Centre for Field Robotics, The University of Sydney, 1999.

[8] M. Betke and K. Gurvits, "Mobile robot localization using landmarks," 1994. [Online]. Available: citeseer.ifi.unizh.ch/betke94mobile.html

[9] J. O. Wallgrun, Spatial Coginition IV, Autonomous Construction of Hierarchical Voronoi-Based Route Graph Representations, 2005.

[10] M. Garber and M. C. Lin, Algorithmic Foundation of Roboitca V; Constraint-Based Motion Planning Using Voronoi Diagrams. SpringerVerlag Berlin, 2004.

[11] Whiley, "Journal of graph theory," http://www3.interscience.wiley.com/cgi-bin/jhome/35334, Jan 2006.

[12] N. Rao, S. Kareti, W. Shi, and S. Iyenagar, "Robot navigation in unknown terrains: Introductory survey of non-heuristic algorithms," 1993. [Online]. Available: citeseer.ifi.unizh.ch/rao93robot.html

[13] A. V. Goldberg and C. Harrelson, "Computing the shortest path: A* search meets graph theory," in 16th Аnпиal ACM-SIAM Symposium on Discrete Algorithms (SODA '05), Vancouver, Canada, 2005.

[14] Wikipedia-The_Free_Encylopedia, "Mahalanobis distance," Feb 2006. [Online]. Available: http://en.wikipedia.org/wiki/Mahalanobis_distance

[15] S. E. Rönnbäck, "The circle sector expansion method," July 2006. [Online]. Available: http://mica.csee.ltu.se/cse

[16] Wikipedia, "de casteljau's algorithm," May 2006. [Online]. Available: http://en.wikipedia.org/wiki/De_Casteljau's_algorithm

[17] S. Rönnbäck, D. Rosendahl, and K. Hyyppä, "A matlab/java interface to the mica wheelchair," The 1st IFAC Symposium on Telematics Applications in Automation and Robotics, pp. -, July 2004.

[18] SICK, "Sick and lms200 and laser measurement system," http://www.sick.com, Dec 2003.

[19] OSG_Community, "Open scene graph,” May 2005. [Online]. Available: http://www.openscenegraph.org/ 